\title{
HACIA UN MODELO DE INCLUSIÓN SOCIAL MEDIANTE LA INTERVENCIÓN SOCIOEDUCATIVA Y ECONÓMICA
}

\begin{abstract}
Resumen
En este artículo se expone un modelo de intervención socioeducativa y económica cuyo fin es la inclusión social y se presenta como respuesta al nuevo fenómeno de la exclusión aparecido en las sociedades de los llamados países desarrollados. Este modelo se ha aplicado en una experiencia llevada a cabo en España durante el año 2006, en distintas barriadas de la Comunidad Autónoma de Andalucia, detectadas como áreas de riesgo para la exclusión social de jóvenes, que debido a circunstancias de tipo social, económico, familiar y formativo, se encontraban en situación de riesgo de exclusión social. Dicha intervención, se basa en la idea de que el trabajo como actividad social porta factores de crecimiento personal siendo el empleo un vehículo privilegiado para la inclusión. Sin embargo, la realización del primero o la consecución del segundo, requieren la concurrencia coordinada de agentes como la sociedad civil y la administración pública más allá del asistencialismo, así como de estrategias de carácter educativo.
\end{abstract}

Palabras clave: Intervención socioeducativa, inclusión social, exclusión social, modelo tecnológico, asistencialismo, inserción laboral.

\section{TO A MODEL OF SOCIAL INCLUSION THROUGH THE SOCIAL-EDUCATIVE AND ECONOMIC INTERVENTION}

\section{Abstract}

This article proposal shows a model of intervention aiming at social inclusion and targets those vulnerable groups set in the societies of the so called developed countries. The experience took place in Andalucia (Spain) during the year 2006, in different excluded areas (slums). Youngsters of these districts were considered to be at risk of exclusion due to their social economic realities, family environment and lack of adequate training.

* Doctor en Pedagogía. Profesor de la Universidad de Granada (España). gcarmona@ugr.es 
The intervention mentioned above, is based on the employment regarded as a social activity that has a clear effect in personal growth and is thereafter a key inclusive element. Nevertheless, either employment or social inclusion need the performance of civil society, public administration and of tools like programs and projects of intervention using social and educational strategies allowing to reach the "real citizenship" equal to those considered socially included.

The experience has been developed in the frame of the Plan de Intervención contra la Exclusión Social en Zonas (barriadas) de Actuación Preferente, a program that aims at social inclusion run by the Regional Government Junta de Andalucia

Keywords: Intervention associate educational, citizenship, social inclusion, social exclusion, labour insertion, welfare, technological model.

\section{INTRODUCCIÓN}

En los últimos años, la metodologia de la intervención social ha experimentado múltiples cambios, tanto en el orden teórico como en el práctico, para adaptarse a la pluralidad de las nuevas realidades sociales que, caracterizadas por la complejidad, dinamicidad e interacción de los elementos que las conforman, han dado lugar a situaciones variadas con desigual impacto en la vida de las personas, lo cual exige respuestas ajustadas.

En este artículo se propone un modelo de intervención cuyo fin es la inclusión social y se presenta como respuesta al nuevo fenómeno de la exclusión que ha aparecido en las sociedades de los llamados países desarrollados. Este modelo se ha aplicado en una experiencia llevada a cabo en España durante el año 2006, en distintas barriadas de las ciudades de Sevilla y Granada en la Comunidad Autónoma de Andalucia, detectadas como áreas de riesgo para la exclusión social de jóvenes, que debido a circunstancias de tipo social, económico, familiar y formativo, se encontraban en situación de riesgo de exclusión social.

Dicha intervención descansa en la idea de que el trabajo como actividad social porta factores de crecimiento personal siendo el empleo un vehículo privilegiado para la inclusión. Sin embargo, la realización del primero o la consecución del segundo, requieren la concurrencia de agentes como la sociedad civil y la administración pública, así como de ins- trumentos como programas y proyectos de intervención que basados en estrategias de carácter socioeducativo, permitan alcanzar el grado de ciudadanía de hecho característico de las personas consideradas socialmente incluidas. La experiencia aludida se encuadra en un programa que forma parte del Plan de Intervención contra la Exclusión Social en Zonas (barriadas) de Actuación Preferente, dependiente de la Consejería de Asuntos Sociales de la Junta de Andalucía.

\section{MARCO TEÓRICO Y FUNDAMENTACIÓN: HACIA LA SUPERACIÓN DEL MODELO TECNOLÓGICO}

Los cambios sociales han obligado a un replanteamiento de los modelos de intervención social adoptados durante la época del desarrollo industrial de los años 70 . La fundamentación de dichos modelos es criticada por responder a una sociología funcionalista de la que se desprenden propuestas exclusivamente de tipo asistencial, las cuales dan lugar a formatos de intervención social de espera, de atención en ventanilla con escasa atención individualizada, insatisfactorios para el logro de mejoras significativas en las comunidades (Bueno Abad, R. 1991).

Como consecuencia del debilitamiento de los postulados del modelo tecnológico surgen alternativas que reorientan el protagonismo de las personas, grupos y comunidades desde su representación formal a su actuación real (Caride, J., 1999) haciendo que sean congruentes con la acción comunicativa y dialógica que autores como Habermas o Freire postulan para toda dinámica de intervención social.

Desde esta perspectiva, las personas, las comunidades y los grupos se convierten en actores de cambio, con capacidad para crear y rehacer significados a partir de su realidad, de pensar y decidir en función de su experiencia cotidiana, pasando de considerarse objetos de la atención a ser sujetos de la acción.

Por ello, en un proceso de intervención social auspiciado por esta nueva óptica, los métodos y procedimientos que se utilicen tendrán un papel relevante, desde la exploración inicial de la realidad hasta la formulación y valoración de las acciones orientadas a la transformación y mejora a partir de problemas prácticos. Así, la intervención social no puede reducirse a un proceso de gestión tecnológica, porque a partir de lo expuesto y en opinión de Ponce de León (1985), el objetivo esencial es lograr un cambio de actitud de las personas, afianzando una visión crítica de su problemática, favoreciendo comunidades cada vez más autónomas, que sean capaces de desarrollar su potencial, asumir responsabilidades y exigir sus derechos

Es por esto que los proyectos para la inclusión social, se apoyan en la actualidad en esta nueva concepción que está siendo adoptada en el ámbito del trabajo social y en la sociología de la exclusión, porque en gran medida reformula la expresión lucha contra la exclusión social que denota una concepto asis- 


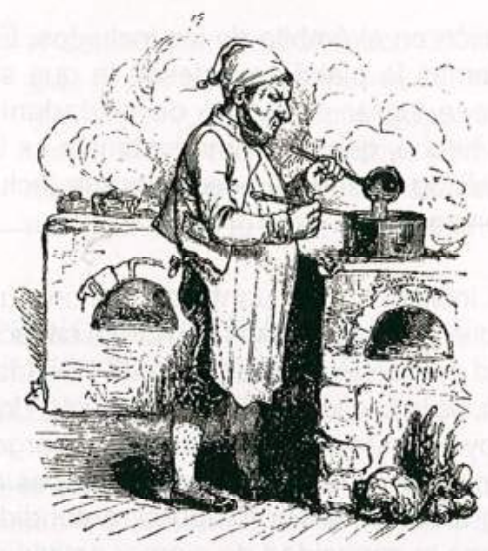

tencialista y, sobre todo, porque innova en la perspectiva de actuaciones contra la exclusión.

En efecto, tradicionalmente las acciones dirigidas contra la exclusión social vienen figurando de manera casi exclusiva dentro de los Planes de Empleo, siguiendo el citado modelo tecnológico. $\mathrm{Y}$ cuando no, aparecen atomizadas y discurren a través de múltiples unidades u organismos administrativos de forma poco coordinada.

El cambio de perspectiva que proponemos se asienta sobre tres pilares fundamentales: 1. la necesidad de una mejor comprensión del fenómeno de la exclusión social por parte de los gobiernos $y$ administraciones, hasta ahora principales protagonistas en la elaboración y diseño de programas; 2 . en el carácter integral, coordinado y corresponsable de las acciones a realizar por parte de la Administración Pública, 3. en la incorporación colaboradora y activa de los agentes de la administración local, sociedad civil y de los propios grupos y comunidades afectadas.

\section{COMPRENSIÓN DEL FENÓMENO DE LA EXCLUSIÓN SOCIAL}

Sobre la exclusión social en el Plan Nacional de Acción para la Inclusión social del Reino de España (Ministerio de Trabajo y Asuntos Sociales, 2003), se señala el potencial descriptivo y el valor para el análisis de la noción "exclusión social". En ella destacamos cuatro elementos clave que nos van a permitir una mejor comprensión de las dimensiones del fenómeno en las distintas realidades, por lo que a continuación las exponemos:

\section{Dimensión estructural}

Las dinámicas y situaciones de marginación que afectan hoy a ciertos colectivos se instalan en la trayectoria histórica de las desigualdades sociales, las cuales en su día dieron lugar a la "cuestión social", que en la actualidad, se transforma y adquiere una naturaleza nueva en la sociedad postindustrial. En la sociedad industrial las desigualdades se centraban generalmente en la dimensión económica, asunto que no llegaba a romper los parámetros básicos de la integración social. Sin embargo, la exclusión social implica fracturas en el tejido social y la ruptura de las coordenadas básicas de cohesión. Esta escisión se produce dentro y fuera de la organización social y no de mejor o peor posición económica en la estructura.

\section{Dimensión dinámica}

La exclusión se produce mediante un conjunto de procesos motivados por la erosión y el debilitamiento de los soportes de seguridad que la modernidad industrial ha generado y que afectan de forma variable a personas y colectivos siendo consecuencia de ello la separación y la dificultad de acceso al ejercicio de los derechos básicos de la ciudadanía. En este contexto, la distribución de riesgos sociales, la convierte en aleatoria y de extensión indeterminable. Las fronteras de la exclusión son móviles; pues los índices de riesgo de ser afectado por ella alcanzan extensiones sociales e intensidades personales cambiantes.

\section{a. Dimensión multifactorial y multidimensional}

La exclusión se explica por la incidencia de un conjunto de factores entre los que encontramos la fragmentación social, la crisis del empleo y/o las limitaciones de las políticas de bienestar. La multidimensionalidad se muestra como un fenómeno poliédrico que afecta a las esferas de lo personal, familiar, social, económico, la salud y que está conformado por la articulación de circunstancias desfavorables en estos aspectos que a menudo aparecen fuertemente interrelacionados.

\section{b. Dimensión estratégica}

En la sociedad industrial las personas generaban capacidad de autoorganización a partir de la comprensión de su situación, como propietarios de medios de producción o como operarios en los mismos. Esto no ocurre con la exclusión, pues los excluidos no conforman un sujeto homogéneo y articulado de cambio histórico, como era el caso del movimiento obrero, ni visible y con capacidad de superación de su situación de exclusión. De ahí que sea mucho más complicado generar procesos de movilización y definir una praxis emancipatoria. Por ello, se suele ver la exclusión desde una perspectiva cultural que nos lleva a considerarla como algo inherente a las sociedades avanzadas del siglo XXI. También se intenta reducir la exclusión a un problema de falta de capacidad individual para seguir el ritmo del conjunto de la sociedad.

\section{CARÁCTER INTEGRAL Y COORDINADO DE LAS INTERVENCIONES A REALIZAR POR LAS ADMINISTRACIONES PÚBLICAS}

El trabajo en la sociedad industrial actuaba como eje central de la vida social y por tanto, las políticas de empleo primaban sobre cualquier otra. Hoy, este criterio está siendo revisado por aquellos teóricos y prácticos que afirman que el puesto central que ocupaba el trabajo, está siendo sustituido por el consumo como fundamento de la integración social (Rivero, A., 2000). El cambio de óptica que aporta esta última tesis es sustancial, por cuanto descentra del trabajo las acciones contra la exclusión social. Esta nueva visión remite a la necesidad de actuar sobre distintas áreas 
de lo social y lo personal que, hasta ahora, no se habian tenido en consideración y que, sobre todo, estaban desconectadas entre sí de cara a la lucha contra la exclusión. Así, la intervención para la inserción laboral consistía en acciones dirigidas a personas o comunidades tendentes a la capacitación laboral basadas en la Formación Profesional Ocupacional, sin considerar la modalidad de tales acciones en función de circunstancias como la salud, las adicciones, la precariedad vital, etcétera.

\section{COLABORACIÓN ACTIVA ENTRE LA ADMINISTRACIÓN LOCAL, LA SOCIEDAD CIVIL Y LOS GRUPOS DE AFECTADOS}

La necesaria implicación entre la sociedad civil y los grupos de afectados se justifica en que los resultados obtenidos a partir de las acciones cogestionadas entre agentes sociales y administraciones locales son más eficientes $y$, eficaces, dada su cercanía al ciudadano (Plan Nacional de Acción para la Inclusión Social, 2003). La integración social está intimamente ligada a la posición que el individuo ocupa en el mercado laboral, éste se ubica fundamentalmente en el ámbito de lo privado y la exclusión del mismo supone además la pérdida de los mecanismos de protección social asociados al empleo. Por tanto, se hace necesaria la interacción entre las distintas agencias de la sociedad civil, para abrir nuevamente las vías de inclusión a las personas o grupos en riesgo social. Todo esto sostiene, en buena medida, que se utilice la expresión Inserción social mediante el empleo o Inclusión social por lo económico. Estos términos hacen referencia al conjunto de prácticas públicas y privadas que tratan de cooperar con los mecanismos ordinarios de integración social y ofrecen oportunidades directas o indirectas de acceso al empleo a aquéllas personas cuya participación en el mercado laboral sin estos apoyos sería difícil.

Como hemos visto, las políticas de integración hasta los años 80 , buscaban la cohesión de la sociedad mediante su homogenización a partir de factores como el trabajo (condición salarial). Las medidas de inclusión que proponemos suponen una discriminación positiva centrada sobre poblaciones especiales y zonas determinadas del espacio social.

El cambio de perspectiva con respecto al problema de la exclusión social es de gran calado, pues supone un desplazamiento de la responsabilidad. Si antes era la Administración en exclusiva quien tenía que articular las medidas para la integración, ahora, desde la perspectiva de la inclusión, es la colaboración entre la sociedad civil y la administración quienes situando a la persona en el núcleo de su intervención, lograrán realizar en ella las transformaciones que le permitan insertarse en la sociedad.

La tesis sobre la cual se va a fundamentar la política de inclusión consiste en que los entornos de producción material y simbólica se están haciendo cada vez más restringidos y competitivos. Esto supone que cada vez más personas quedan alejadas del ámbito de concurrencia laboral $y$, por ende, con menos posibilidades reales de obtener empleo estable (Soriano, A, 2001). Consecuentemente, el objetivo que se persigue es fomentar el desarrollo de oportunidades mediante la formación, la orientación y el acompañamiento social para conseguir mayores garantías de acceso a la integración social y al trabajo.

\section{PRINCIPIOS Y JUSTIFICACIÓN DEL MODELO DE INCLUSIÓN SOCIAL MEDIANTE LA INTERVENCIÓN SOCIOEDUCATIVA Y ECONÓMICA}

Según lo expuesto, la inclusión social se podria representar como un itinerario particular que han de transitar las personas o grupos excluidos y en el que deberian alcanzar las condiciones necesarias para trasladarse de fuera a dentro del sistema social.

La inserción laboral es el factor más relevante, aunque no el único, en el proceso de inclusión, pues permite a las personas obtener autonomía económica y sociali- zación en el ámbito de los incluidos. Es además la plataforma desde la que se puede lograr el estado de ciudadanía de hecho, que en última instancia es la finalidad de todo proceso para la inclusión social.

La inclusión, en cuanto expresión que representa a personas con plena capacidad de ejercer sus derechos de ciudadanía, es la finalidad que se persigue en los proyectos de intervención, sin embargo, el objetivo que éstos se proponen es el logro de la inserción laboral, entendida como la capacidad de ejercer actividad profesional en un mercado de trabajo. Convertir en empleables a las personas destinatarias de tales proyectos será el objeto del diseño y de las distintas acciones a realizar, tarea que exige conjugar el conocimiento del medio socioeconómico de destino (mercado laboral) y el conocimiento del entorno social y personal de los participantes.

El logro de la empleabilidad consiste en que las personas alcancen el plus de capacidad para situarse en el mercado de trabajo en condiciones de ser contratadas, esto se traduce en hacerlas pasar por distintos dispositivos de formación, orientación e inserción, articulados en una sucesión de etapas cuya superación conduce al desarrollo de sus competencias para entrar en la categoria de empleable (Eme, B., 1997).

Esto supone la formación para el aprendizaje de conceptos, hechos, normas, valores y actitudes orientados tanto a la mejora de la educación personal, como a la socialización y a la adquisición de conocimientos prácticos profesionales.

\section{Esta formación va dirigida:}

a. a personas, de manera que se incida especialmente en la adaptación individual a las exigencias del medio productivo.

b. a grupos de personas, donde el enfoque de la acción pedagógica tiene como objetivo realizar una socialización funcional que implique efectos de apertura y motivación al aprendizaje. 
c. al contexto socioeconómico de acogida de los excluidos, donde la transformación funcional, que supone la condición de los aprendizajes individuales y las formas de socialización productiva, es lo más importante.

\section{PRINCIPIOS GENERALES DE NUESTRA INTERVENCIÓN SOCIOEDUCATIVA}

El análisis de necesidades de los potenciales destinatarios de la intervención socioeducativa revela que tienden a establecer la marginalidad como sistema de vida, debido a que la búsqueda de recursos para la subsistencia ocupa la totalidad de su tiempo y organiza sus vidas, tanto en la dimensión social como en la privada.

La escasa atención prestada a la promoción personal se convierte en un agente causal de marginación de primer orden, lo que hace necesaria la intervención justamente sobre esta variable.

La formación y promoción social, así como cualquier otra forma de actividad social que no conduzca de forma directa a la subsistencia será considerada secundaria. El desarrollo personal, desde nuestro punto de vista, requiere de la mejora de las cualidades y las potencialidades atendiendo a tres planos fundamentales:

a. Cognitivo: conocimientos generales y saber técnico, saber hacer.

b. Socioafectivo - emocional: saber ser y actitudes positivas hacia el trabajo.

c. Comportamental: saber estar.

Esto significa que la intervención debe ser de carácter integral y tiene que incidir en las tres áreas básicas señaladas. Consecuentemente los principios y elementos necesarios para el proceso del modelo de inclusión mediante formación para la inserción laboral son los siguientes:

- Coordinación. Entre administraciones públicas, entidades privadas (fundaciones, ONG), iniciativas sociales (movimiento asociativo, economía

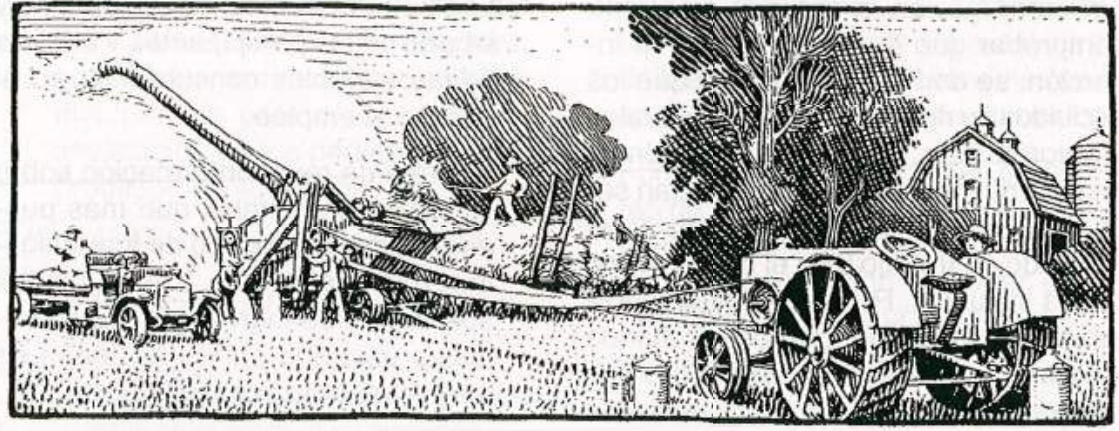

social) y las empresas, involucradas en la inclusión social.

- Integralidad. Que permita el desarrollo de todas las potencialidades de la persona y actúe sobre todos los ámbitos sociopersonales necesarios para la consecución de los objetivos propuestos.

- Participación activa. Que haga posible la superación del modelo asistencial impulsando, a su vez, dinámicas de implicación de los usuarios, de sus familias y de entorno en la gestión, conocimiento y coordinación con los agentes sociales y las Instituciones en su actuación contra la exclusión.

- Reflexión-planificación-acción-reflexión. La intervención exige la reflexión conjunta de los actores concernidos, acerca de los fines objetivos, medios, recursos y estrategias a seguir, para lo cual se seguirá la secuencia de acciones expuesta.

- Intervención personalizada e individualizada. Las actuaciones tienen como fin la promoción personal orientada al ejercicio real de la ciudadanía. Para ello se proponen como objetivos la atención individual y la formación personalizada de los usuarios durante el desarrollo de proyectos para la integración social.

- Interdisciplinariedad. Es una condición necesaria que abarca al trabajo social y que impregna la intervención, posibilitando el abordaje de las problemáticas derivadas de la exclusión, caracterizadas por su multifacialidad.

- Aprender trabajando y trabajar aprendiendo. La inclusión social pasa por la inserción laboral y para el logro de ésta será fundamental la experiencia de trabajar. Los distintos aspectos a tratar (social, personal, económico) en los usuarios, se abordarán desde la inmersión en la cultura de lo laboral y el empleo

\section{DISEÑO DE LA INTERVENCIÓN SOCIOEDUCATIVA PARA LA INCLUSION SOCIAL Y ECONÓMICA}

Entendemos por Diseño la estructura de acciones educativas de orientación profesional y de dinamización socioeconómica encaminadas a la consecución de los objetivos establecidos mediante la adecuada organización de las mismas. El diseño es previo a la ejecución de la intervención y condiciona la realización de la misma, por lo que es conveniente observar la zona de actuación para comprender las relaciones y factores que configuran el escenario donde se ha de actuar.

La planificación requiere de la permanencia previa en el contexto de actuación con el fin de obtener información de personas clave, agentes comprometidos con el trabajo social en la zona. Esto permitirá conocer para actuar sobre los factores y relaciones que dan origen a la situación y necesidades de los destinatarios de la intervención.

Hasta ahora, los planes para inserción laboral han incidido en el desarrollo de la capacitación técnica de los usuarios, mediante el desarrollo de habilidades laborales, así como en los incentivos a la contratación como medida de apoyo. 
Sin embargo, en la realidad se puede comprobar que la dificultad para la inserción, se deriva del hecho de que los excluidos se rigen por criterios culturales y valores para lograr la sobrevivencia en el territorio marginal que resultan ser contrarios a los que predominan en el mercado de trabajo y en el medio social de los incluidos. Por tanto, los comportamientos consecuentes con los citados valores se convierten en prácticas disruptivas en el orden laboral, y en la vida social ligada al trabajo.

Ante esto, la capacitación técnica es un elemento casi de segundo orden, por lo que se impone un trabajo educativo mediante el cual el sujeto afectado por la exclusión pueda reconstruir su visión del mundo y esto supone afrontar un proceso de reorganización personal, que implica modificación en lo cognitivo en lo afectivo-emocional y en el comportamiento.

Este planteamiento exige realizar un diseño de la intervención basado en lo educativo y también en función de los objetivos y metas que marcan las necesidades para la inserción laboral de cada uno de los usuarios. Por ello, hemos de considerar proporcionalmente y en el mismo orden de importancia el tiempo y el espacio de dedicación necesario a la cualificación técnica y a la mejora personal y social; se tendrán en cuenta los ámbitos de actuación: personal, socioeducativo y socioeconómico.

A continuación se exponen los campos de intervención y la secuencia de acciones a realizar.

\section{Ámbito personal}

En este espacio se llevan a cabo todos aquellos procesos que inciden en el progreso personal de cada participante, fomentando sus habilidades sociales y una educación dirigida al reconocimiento de las propias potencialidades: la formación, la cualificación profesional y la experiencia laboral.

Para ello se realiza el siguiente protocolo de actuaciones:
- Examen del currículum académico y laboral de los participantes y análisis de las variables concurrentes en el acceso al empleo.

Se trata de reunir información sobre los datos personales que más pueden afectar al proceso de formacióninserción laboral a través de las solicitudes de ingreso de los potenciales usuarios en las acciones de proyectos para la inclusión social.

Estos aspectos, que tras ser valorados aportan el grado de potencial empleabilidad de los sujetos analizados son:

a. Potencialidades personales (motivación, intereses, valores)-

b. Potencialidades profesionales (conocimiento de oficios, habilidades, destrezas e intereses profesionales).

c. Factores inherentes a la inserción laboral (cultura de empleo, conocimiento del mercado laboral).

d. Problemática social general (salud, vivienda, formación, cultura, familia, justicia).

\section{- Diagnóstico.}

Con posterioridad al análisis del grado de presencia de los factores personales, técnico- cognitivos y formativos para el empleo, debe realizarse una evaluación previa de la situación sociopersonal de los usuarios, con el fin de orientar las actuaciones de intervención sociofamiliar, acompañamiento social, de formación y orientación profesional, que se realizarán posteriormente para incrementar su nivel de empleabilidad.

- Itinerario individualizado y personal para la inserción laboral.

A partir de las cuatro variables señaladas como más relevantes para la inserción laboral (personales, profesionales, cultura de empleo, problemática social) deben implementarse las siguientes acciones, según el grado de necesidad detectado:

- Orientación para el empleo y la inserción sociolaboral que incluye acciones para:
- Desarrollo de habilidades personales.

- Desarrollo de habilidades sociales.

- Información y formación para búsqueda activa de empleo.

- Asesoramiento profesional.

- Formación dirigida a cubrir aspectos deficitarios detectados:

- Formación profesional ocupacional en oficios con alta demanda en el mercado laboral.

- Formación reglada compensatoria.

- Formación en actitudes y valores para el trabajo.

- Gestión de la inserción laboral dirigida a dos planos de actividad económica:

- Por cuenta ajena, para la que planteamos como estrategia de incorporación a la empresa las siguientes posibilidades:

- Periodo de formación-producción en empresas normalizadas.

- Periodo de formación-producción en empresas de inserción.

- Unidades de producción en empresas de inserción.

- Autoempleo o creación de empresas, cuya estrategia para la incorporación de usuarios puede realizarse (según el caso) a través de las siguientes vías:

- Empresa con la figura de FormadorGerente.

- Empresa asociada a una empresa de inserción.

- Empresa con un agente externo emprendedor.

- Consolidación del puesto de trabajo y/o de la empresa:

Este aspecto de la gestión para la inserción laboral de excluidos se incorpora al modelo de intervención, habida cuenta de las dificultades encontradas en la práctica. Por lo que se recomienda tomar en cuenta los siguientes extremos: 
- En el caso de creación de empresa, realizar el plan estratégico de consolidación de forma participativa, entre promotores de la misma y usuarios de proyectos de intervención.

- Implicar de forma colaborativa en el proceso a entidades de iniciativa social, ONG y administración que actúan en el territorio de referencia.

- Captar y consolidar mercados de producción.

- Formar mediante el subsistema de formación continua, a promotores emprendedores y a usuarios, cada cual en las funciones que vaya a desempenar.

\section{INTERVENCIÓN SOCIOEDUCATIVA}

Como se ha expuesto, la inserción laboral de los excluidos requiere de la integralidad en la intervención, por lo que orientaremos buena parte de las actuaciones al plano sociopersonal.

- Diseño del plan de intervención socioeducativo.

Será necesario realizar un análisis de las necesidades socioeducativas de cada uno de los participantes que permita detectar las problemáticas económicas, sociales, comunitarias, familiares, de vivienda, salud y de alfabetización digital existentes. De acuerdo con este análisis se diseñará un plan de intervención personalizado y adaptado a cada uno de los participantes.

En este proceso, deben seguirse los siguientes pasos:

- Analizar y priorizar de las problemáticas familiares detectadas.

- Contactar con los Servicios Sociales para establecer vías de coordinación y, en su caso, derivación de sujetos para que reciban la atención correspondiente.

- Establecer planes de intervención simultáneos sobre los sujetos y sus familias tomando en cuenta sus circunstancias.
- Diseñar, en coordinación con los Servicios Sociales Comunitarios, actividades de tipo lúdico, cultural, divulgativas para el desarrollo e implicación de los participantes en procesos comunitarios:

- Actividades Lúdicas y culturales.

- Actividades de divulgación y sensibilización.

- Actividades de implicación de la familia en el proceso de inserción

\section{INTERVENCIÓN SOCIOFORMATIVA}

La educación permanente o de apoyo educativo tiene como finalidad compensar los déficits educativos de los participantes, con objeto de mejorar el aprovechamiento de la formación profesional y permitir que obtengan una titulación académica reglada.

Desde el inicio de las intervenciones de inserción laboral es necesario articular e incluir acciones de apoyo educativo. Será preciso, por tanto, establecer convenios de colaboración con la administración educativa para que desarrollen esta intervención y favorezcan la obtención del título de enseñanza básica obligatorio, adaptando los medios de la educación formal a las especificidades de las personas marginadas que están participando en acciones de formación para la inserción laboral.

\section{INTERVENCIÓN SOBRE EL ENTORNO SOCIOECONÓMICO}

Las acciones que deben llevarse a cabo se dirigen al logro de objetivos coincidentes con los que señala el profesor Ander-Egg (1967) para el desarrollo comunitario, a saber:

- Aumentar el conocimiento sobre las fuerzas económicas, sociales y politicas que explican y provocan la existencia de la pobreza, la desigualdad, la opresión y que condicionan nuestras vidas como sujetos pertenecientes a cualquier cultura del planeta.
- Desarrollar valores, actitudes y destrezas que acrecienten la autoestima de las personas, capacitándolas para ser más responsables de sus actos.

- Fomentar la participación en propuestas de cambio para lograr un mundo más justo en el que tanto los recursos y los bienes, como el poder estén distribuidos de forma equitativa.

- Dotar a las personas y a los colectivos de recursos e instrumentos cognitivos, afectivos y actitudinales que les permitan incidir en la realidad para transformar sus aspectos más negativos.

- Favorecer el desarrollo humano sostenible en los tres niveles que afectan a las personas: individual, comunitariolocal e internacional.

Por ello, la intervención se compone de acciones de carácter educativo, con referencias macroestructurales y propósitos de desarrollo económico, cultural y personal. Para lo cual, en nuestro modelo seguimos las siguientes pautas:

- Informar e implicar a la familia de los usuarios en el proceso de intervención, fomentando tanto su apoyo individual, como su organización colectiva para favorecer las condiciones (recursos y medios) de las acciones de la intervención.

- Fomentar el asociacionismo en torno a las problemáticas de desempleo para poder ejercer medidas de presión ante la sociedad, la administración correspondiente y los poderes públicos

- Dinamizar la zona donde se están desarrollando los proyectos de intervención socioeconómica y educativa, y promover el desarrollo económico, social y comunitario de las mismas.

Esta actuación se asienta en el principio pedagógico de "ir de lo particular a lo general", para ello se pretende facilitar la comprensión del mundo a los usuarios participantes de proyectos de inclusión social a partir de la interacción con él. Por ello, este apartado de la intervención requiere de las siguientes pautas especificas: 
- Detectar nuevos yacimientos de empleo, mercado productivo y análisis de viabilidad para proyectos de creación de nuevas empresas.

- Colaborar con los agentes económicos públicos y privados de la zona en la que se interviene para que se favorezca la inserción de estos colectivos.

- Establecer convenios y compromisos de contratación con empresas ligados a acciones previas de formación profesional.

- Articular compromisos con las administraciones públicas locales para desarrollar la contratación de obras y/o servicios a partir de los que iniciar empresas con los usuarios participantes en la intervención.

- Desarrollar relaciones comerciales y canales de distribución que favorezcan la producción y consolidación de las empresas creadas.

- Favorecer el establecimiento de relaciones y redes de colaboración y cogestión entre entidades, instituciones y empresas que trabajan desde la perspectiva de la inserción social.

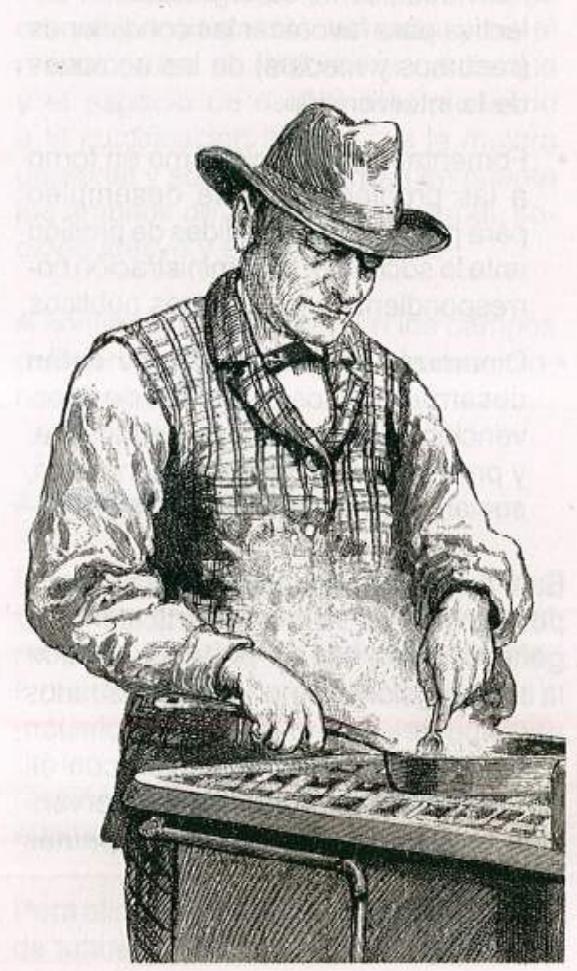

\section{METODOLOGÍA}

La metodología empleada en este modelo de intervención es de tipo investigación-acción. La puesta en marcha de un proyecto dentro de este marco metodológico exige el conocimiento preciso de las necesidades de las personas a las que se dirige, la coordinación con instituciones que intervienen en la zona y, sobre todo, la participación activa de los propios beneficiarios.

Los objetivos son el resultado de la selección y jerarquización de las necesidades expresadas por los actores y participantes. Las acciones se plantean como medios para lograr los objetivos y son de carácter práctico, similares a las utilizadas por las Escuelas de Formación acelerada de California (Ayuste, A., 1994), en las que predomina el aprendizaje significativo y los contenidos orientados a la práctica inmediata, organizados de tal manera que se apoyan mutuamente y se refuerzan para lograr cambios actitudinales en los alumnos.

La practicidad, unida a la inmediatez de respuesta a las necesidades vitales del usuario, se convierte en un elemento motivacional de primer orden, de tal manera que perciben la coherencia entre organización de acciones, contenidos y objetivos para lograr los fines de la intervención, y esto hace que se produzca la participación y cogestión en la misma.

Por otra parte, los profesionales de la educación social actúan en el desarrollo del proyecto bajo el principio de interdisciplinariedad, de ahi que las áreas de intervención estén interrelacionadas y coordinadas, consiguiendo así un flujo permanente de información mutua que facilita la reflexión sobre la acción.

La evaluación final del proyecto de intervención se hace de manera conjunta y en la cual participan los distintos estamentos involucrados (educadores sociales, usuarios y entidades colaboradoras). Es el momento en el que se decide qué aspectos del diseño inicial se han de modificar para poner en marcha una nueva planificación acorde con los problemas detectados durante la implementación del plan derivado del diseño inicial, generando así una espiral autoreflexiva consistente en:

- Reflexión. Conocimiento inicial del ámbito de actuación que da lugar al diseño general de la intervención y al primer plan de acción.

- Acción. Puesta en marcha e implementación del primer plan de intervención.

- Reflexión. La evaluación del primer plan se fundamenta en la crítica de la propia actuación y en la consiguiento reflexión para mejorar la intervención mediante la elaboración de un segundo plan.

- Acción. Puesta en marcha e implementación del segundo plan de acción.

Se da lugar así a la cadena secuenciada de reflexión-acción que comporta esta metodologia.

\section{EVALUACIÓN}

En nuestro modelo de intervención socioeducativa y económica, la evaluación se plantea como un proceso continuo que tiene por finalidad conocer la marcha del proyecto, asi como su mejora. El procedimiento utilizado es el análisis de la situación en cada fase de la intervención y desde cada uno de los estamentos intervinientes: alumnos, educadores sociales, monitores y agentes sociales colaboradores. Mediante este sistema tendremos una visión ajustada interna y externa de la evolución del proyecto.

La evaluación contempla dos niveles paralelos de ejecución:

Nivel I: Evaluación interna o autoevaluación realizada por los técnicos del proyecto, los colaboradores y los participantes, respectivamente. (Todas las personas que forman parte del proyecto).

Nivel II: Evaluación externa llevada a cabo por expertos. 
Ambos niveles están enmarcados dentro de una perspectiva formativa de seguimiento, de tal modo que en cada fase del proyecto esté contemplada la retroalimentación sobre el proceso en marcha.

Nivel I: Evaluación Interna o Autoevaluación.

\section{- Evaluación inicial}

Supone la valoración inicial de necesidades. Previa a la ejecución, permite contextualizar la intervención objeto del proyecto mediante un estudio de los factores sociales, económicos, culturales y ambientales que evidencian la falta de respuesta a la problemática que el proyecto pretende abordar.

- Evaluación continua y seguimiento

Implica la observación, día a día, de la evolución del proyecto por parte de los técnicos, colaboradores y participantes en el mismo.

- Evaluación desde los educadores sociales y colaboradores

Se plantea a través de indicadores de medidas cuantitativas y de tipo cualitativo, siempre adaptados a las distintas acciones del proyecto.

Al finalizar el proceso evaluador debe emitirse un informe final que aglutine cada una de las evaluaciones previas realizadas durante el proyecto (evaluación interna, evaluación externa), sintetizadas a través de conclusiones que sirvan para la valoración global del mismo. Asimismo se establecerán mecanismos de difusión de dicha información, mediante las entidades participantes, con objeto de servir de referencia a las planificaciones siguientes.

\section{RESULTADOS}

A continuación se exponen los resultados obtenidos en la ejecución del proyecto a partir de la valoración de participantes, con el propósito de obtener datos que anticipen las conclusiones del modelo de intervención socioeducativo y económico.

La población sobre la que se interviene está caracterizada por el riesgo de exclusión y se estima según datos del estudio Equal-Andalucía, Estrategias y metodologías (Equal - Andalucía. Estrategias para el empleo, 2005) en el $1 \%$ del total de los habitantes de las zonas con especiales necesidades de transformación social. Lo que en términos absolutos supone unas 5.000 personas.

- Respecto de los objetivos, los técnicos y entidades colaboradoras a través de entrevista de evaluación manifiestan: a. La derivación de usuarios por parte de los servicios de orientación no estaba bien informada por lo que estos no tenian interés en participar, excepto para ocupar un puesto de trabajo, su grado de empleabilidad era muy bajo

b. Los objetivos no se podian llevar a cabo en parte porque no se ajustaban a la realidad de las empresas de inserción.

c. El tiempo aparece como el tercer elemento de peso en el resultado, resulta insuficiente para lograr hacer comprender a los usuarios el sentido de la intervención.

d. El número de usuarios es excesivo para hacer un trabajo individualizado y con la profundidad que requiere.

e. La dedicación a la realización de memorias y documentación para el control de ejecución del proyecto también se incluye como elemento de restricción en la atención a los usuarios.

- Cómo se cumplen los objetivos desde el punto de vista de los usuarios:

a. La voz de éstos ha sido objeto de atención de primer orden en el proyecto, tal como vemos en la encuesta del grado de satisfacción en las acciones, que arroja los siguientes resultados:

TABLA 1: RESULTADOS DE LOS CUESTIONARIOS DE EVALUACIÓN DE ENTIDADES COLABORADORAS

\begin{tabular}{|l|c|c|c|c|c|c|}
\hline & $\begin{array}{c}\text { Nada de } \\
\text { acuerdo }\end{array}$ & $\begin{array}{c}\text { Poco de } \\
\text { acuerdo }\end{array}$ & De acuerdo & $\begin{array}{c}\text { Bastante de } \\
\text { acuerdo }\end{array}$ & $\begin{array}{c}\text { Muy de } \\
\text { acuerdo }\end{array}$ & Si \\
\hline Conocimiento del proyecto & & & & & $100 \%$ \\
\hline Desempeño de la actividad & & & $17 \%$ & $50 \%$ & $33 \%$ & \\
\hline Metodología empleada & & & $33 \%$ & $50 \%$ & $17 \%$ & \\
\hline Recursos dispuestos & & & $33 \%$ & $33 \%$ & & $66 \%$ \\
\hline Implicación de los técnicos & & & $17 \%$ & $17 \%$ & & \\
\hline
\end{tabular}


TABLA 2: RESULTADOS DE LOS CUESTIONARIOS DE EVALUACIÓN POR LOS PARTICIPANTES EN PORCENTAJES

\begin{tabular}{|c|c|c|c|c|c|c|}
\hline & $\begin{array}{l}\text { Nada de } \\
\text { acuerdo }\end{array}$ & $\begin{array}{l}\text { Poco de } \\
\text { acuerdo }\end{array}$ & De acuerdo & $\begin{array}{c}\text { Bastante de } \\
\text { acuerdo }\end{array}$ & $\begin{array}{l}\text { Muy de } \\
\text { acuerdo }\end{array}$ & Si \\
\hline Acciones de orientación & & & $50 \%$ & $41 \%$ & $9 \%$ & \\
\hline Acciones de formación & & $4 \%$ & $23 \%$ & $54 \%$ & $18 \%$ & \\
\hline Acompañamiento social & & & $32 \%$ & $27 \%$ & $41 \%$ & \\
\hline Relación con técnicos (as) & & & $14 \%$ & $32 \%$ & $52 \%$ & \\
\hline Recursos recibidos & $41 \%$ & $41 \%$ & $18 \%$ & & & \\
\hline
\end{tabular}

- ¿Es necesario este proyecto?

La respuesta de técnicos y usuarios no deja lugar a dudas. La orientación de los servicios habituales "no llega" a las personas excluidas o en riesgo de exclusión. En cambio, este modelo de intervención caracterizado por la atención individualizada, la formación, la orientación y el acompañamiento social, es una propuesta ajustada a las necesidades detectadas mediante diagnóstico y se revela como instrumento de valor para estas personas.

- Procesos que pone en marcha.

En general, se puede decir que afectan a lo personal, mejoran el conocimiento de los sujetos acerca

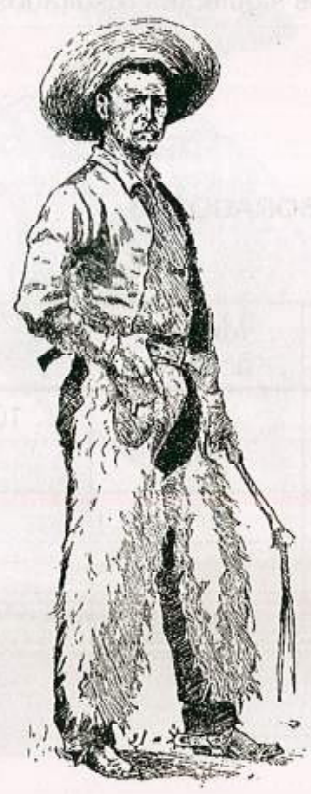

del entorno social "normalizado", movilizan la estructura volitiva, a menudo afectada por las condiciones de desvinculación social a que someten la exclusión y la marginalidad.

También se abren procesos de reconocimiento y comprensión por parte de la sociedad sobre la problemática de las personas excluidas, a través de la difusión y gestión de la contratación en empresas "normalizadas".

- Efectos secundarios

La difusión de los logros del proyecto entre potenciales participantes tiene un efecto positivo, ya que perciben como posible la inserción laboral, si bien es verdad que existe el riesgo de generar expectativas que después no se puedan cumplir, lo que aumentaría el grado de frustración.

\section{CONCLUSIONES}

Los proyectos de inserción sociolaboral desde la nueva perspectiva de la inclusión modifican los modelos asistencialistas o de atención por servicios. En ellos se plantean como objetivos que las acciones de promoción social, formación y empleo de los participantes estén integradas en la dinámica socioeconómica del entorno próximo, ello con el fin de que los usuarios se impliquen en la realidad social. No basta, por tanto, cumplir con las exigencias burocráticas de un servicio administrativo a cambio de percibir algún beneficio económico.
Para ello, se hace necesario el conocimiento, la difusión y puesta en práctica de acciones integradas de promoción social, formación, orientación y empleo entre los propios usuarios, tejido social, económico y los técnicos de los distintos servicios de carácter social de la administración pública, lo que constituye un objetivo en sí mismo.

En este nuevo modelo, el siguiente objetivo será coordinar, en torno al fin propuesto, los recursos de los servicios de la administración pública junto con las entidades privadas de atención social y la red de empresas con responsabilidad social corporativa de la zona.

Así mismo, a partir de la evaluación de proyectos del último año con el modelo de intervención socioeconómica y educativa, es necesario considerar también los siguientes extremos:

1. Realizar una actuación estandarizada y desarrollar una propuesta con la suficiente flexibilidad organizativa que permita en todo momento adecuarse a la realidad del entorno y al colectivo con el cual se trabaja.

2. Es imprescindible la descentralización y territorialización de los servicios y proyectos que ponen en marcha estos procesos flexibles, ya que facilita la colaboración entre entidades y asociaciones (educación de adultos, servicios sociales, centros escolares, asociaciones y familias de los beneficiarios, que tienen que ser partícipes y cómplices desde el comienzo del proceso). 
3. El proceso ha de ser continuo e integral lo que conlleva por un lado:

- Determinar la situación del usuario del proyecto ante el empleo, analizando los factores externos e internos que impiden su inserción y por otro,

- Establecer un itinerario de inserción que implique actuaciones en materia de orientación profesional, formación, inserción laboral y acompañamiento social.
4. El itinerario para la inclusión social es individual y personalizado, elaborado de mutuo acuerdo con el usuario, lo cual implica compromiso y voluntad mutua para llevarlo a cabo. La toma de decisiones es compartida por técnicos, orientadores y usuarios para evitar la dependencia de estos últimos hacia los organizadores de la intervención.
5. Profundizar y analizar exhaustivamente las motivaciones que han provocado la demanda de participación en el proyecto por parte de los usuarios para orientar el sentido de la intervención hacia el fin último de construcción de la autonomia personal.

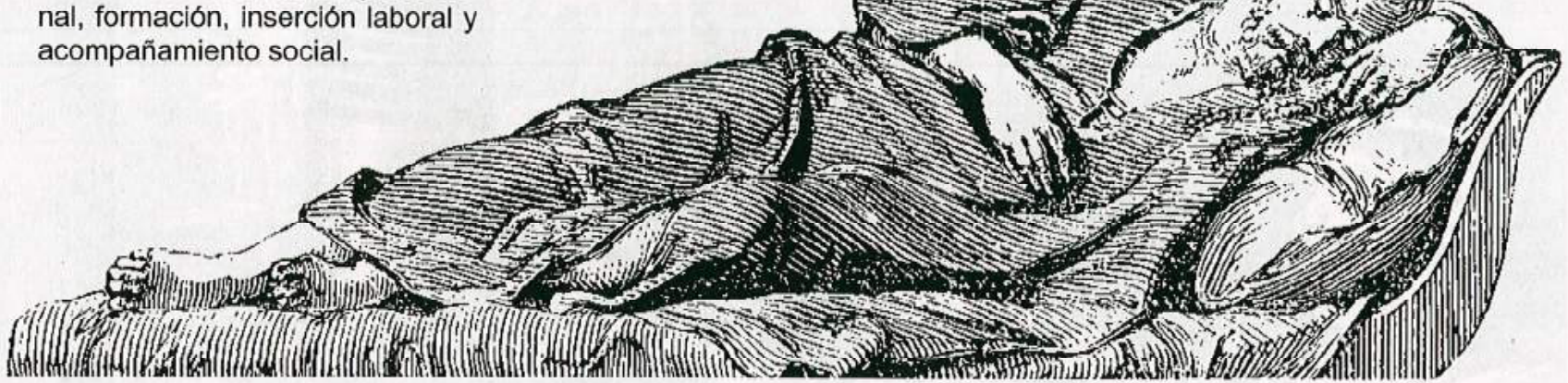

\section{REFERENCIAS}

Ander-EgG, E. (1980). Metodología y práctica del desarrollo de la comunidad. Buenos Aires: El Ateneo.

Ayuste, A. et ál. (1994). Planteamientos de pedagogía crítica. Comunicar y transformar. Barcelona: Graó.

Bueno ABAD, R. (1991). Hacia un modelo de servicios sociales de acción comunitaria. Madrid: Popular.

Caride Gómez, J. A. (1996). La evaluación como mediación. Enfoques y perspectivas para la inclusión social. Revista Perspectiva Educacional. 28, pp. 21-28.
Eme, B. (1997). Órdenes legítimos de participación social y lógicas de cambio social. En Defourny, J. et al. (Dirs). Inserción y nueva economia social. Un balance internacional. Valencia: Ciriec.

Consejería para la igualdad y Bienestar Social (2205). Estrategias y metodologías para la promoción social y económica de zonas con necesidades de transformación social. Sevilla: Consejeria para la igualdad y Bienestar Social.

Ponce de León, G. (1985). Manual de organización y desarrollo para comunidades marginales de las ciudades. México: Trillas
Plan Nacional de Acción para la Inclusión Social del Reino de España. (2003). Madrid: Ministerio de Trabajo y Asuntos Sociales.

Rivero Recuenco, A. (2000). En torno a la exclusión social. Sujetos, predicado e ideología. En Claves de la razón práctica, 18, pp. 39-43.

SORIANo, A. (2001). Políticas y conflictos en la exclusión social. En Pedagogía Social, Revista interuniversitaria de pedagogía

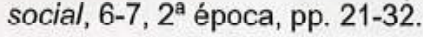

